\title{
Nanoklamotten
}

\author{
Erhard Taverna
}

Elektromagnetische Felder beeinflussen biologische Systeme. Mit 5,8 Millionen Mobilfunkbenützern und 8000 Antennen sowie weiteren Anwendungen in der Medizin, im Arbeitsbereich und im Haushalt gewinnt die Frage nach möglichen Schäden an Brisanz. Handelt es sich beim «Elektrosmog» um ein Phantomrisiko, wenn das Nervensystem mit ähnlichen Frequenzen wie die auf 217 Hertz gepulsten Handysignale arbeitet, wenn die spezifische Absorptionsrate (SAR) ab $20 \mathrm{~mW} / \mathrm{g}$ das Gewebe um 2 Grad Celsius erwärmt, wenn Schlafforscher des USZ bei gesunden Probanden nach Exposition mit gepulster Strahlung eine deutliche, über Stunden erhöhte Hirnaktivität im Nicht-REM-Schlaf nachweisen? Zudem entspricht ein zehnminütiges Gespräch mit Mobiltelefon der Umweltbelastung eines Kilometers Autofahrt.

\section{Die Kontrolleure}

Zwischen Massagesalons, Dancings und Bars versteckt sich das Forschungsinstitut für Informationstechnologie und Gesellschaft (IT'IS) in der Innenstadt von Zürich. Das Nahfeldmesslabor arbeitet eng mit der ETH und der Universität zusammen und wird von einem internationalen Netzwerk aus über 50 Forschungs- und Indu-

SAR distribution in a slice through the body at $450 \mathrm{MHz}$, $900 \mathrm{MHz}$, and $1800 \mathrm{MHz}$ (from left to right).

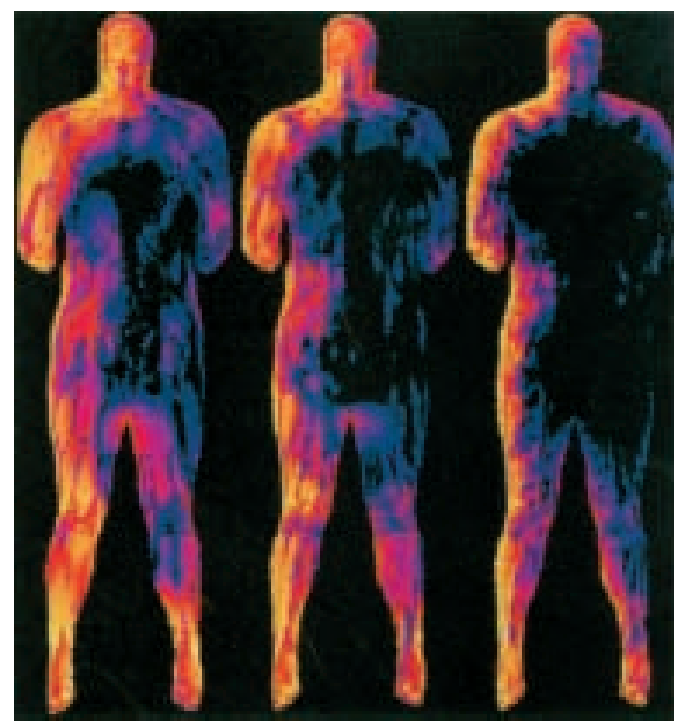

striepartnern getragen. Mehr als 20 aktuelle Forschungsprojekte werden von der EU, von Regierungen, Gesundheitsorganisationen und Fonds der Industrie unterstützt.

Unter der blauen Kuppel abschirmender Polyurethanzapfen versenkt der Roboterarm von Dasy 4 seine Messsonde in eine Zuckerlösung, die das Nervengewebe simuliert. Von unten wird ein Mobiltelefon in variablen Positionen an die schädelförmige Wanne gehalten. Der Computerbildschirm macht die Feldverteilung und die Verlaufskurve der absorbierten Strahlung sichtbar. Praktisch alle Typen von Mobiltelefonen werden hier als farbig kodierte Feinscans dosimetrisch ausgewertet. Zwischen den verschiedenen Modellen sind Abweichungen der Intensität um das 10- bis 20fache möglich. Kritische Punkte seien die Anpassung der Antenne und das Design, erklärt der Student, der hier die Typenprüfungen durchführt. Im Nebenraum werden Werkzeuge entwickelt, die mit numerischen Simulationsmethoden arbeiten. Die Parameter elektromagnetischer Felder werden errechnet und dreidimensional, wie bei der Magnetresonanztomographie, visualisiert. Hier werden Versuchsanordnungen entworfen und unterschiedliche Phantome des menschlichen Körpers ausgewertet. Einen Stock höher sind die Anlagen für Zellkulturen und Tierversuche. In den radiär angeordneten Gehäusen einer Metalltrommel sollen Ratten während zweier Jahre zwei Stunden täglich bestrahlt werden. Eine der grössten toxikologischen Studien zu möglichen karzinogenen Effekten von Mobiltelefonen, an der sich die Schweiz, Österreich und China beteiligen. Hier entstehen die Softwareprogramme und die Expositionsmodelle, ausgeklügelte Anordnungen für In-vivo- und In-vitro-Studien, für Materialprüfungen und Menschenversuche.

Hinweise aus Holland betreffend Kopfschmerzen durch UMTS (universal mobile telecommunication systems) müssen auf Anordnung des BAG überprüft werden. Alle diese Grenzwertkontrollen sind langwierig und kosten. Dasselbe gilt für neue kardiovaskuläre telemetrische Systeme, implantierbare Sensoren zur Überwachung menschlicher Vitalfunktionen oder neue Bioprothesen, die energiearm, aber körpernahe, noch unbekannte Nebenwirkungen auslösen können. 
Head phantoms: a) three-year-old child, b) European female, c) adult male, d) SAM.

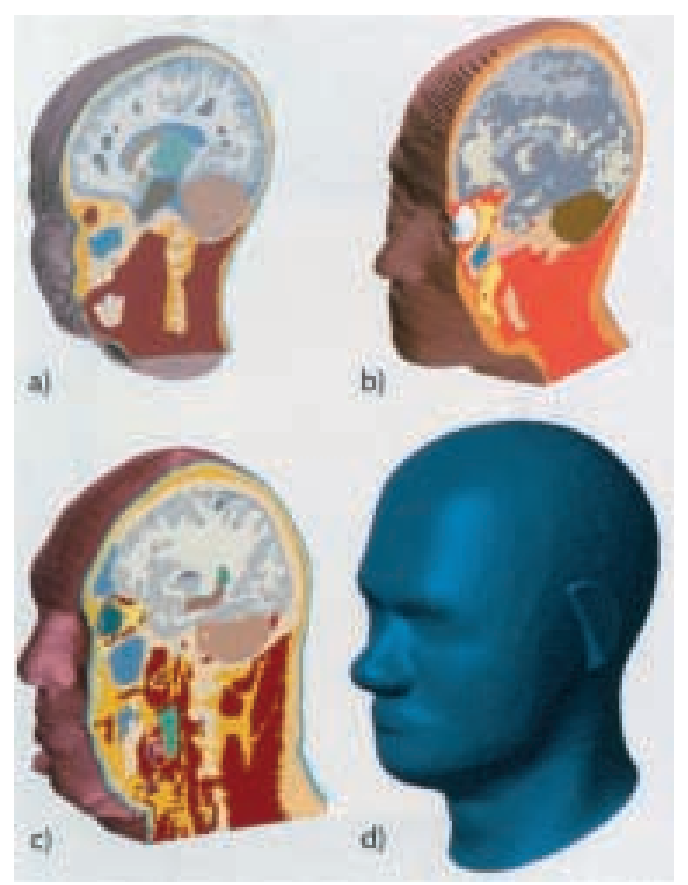

\section{Die Erfinder}

Der Professor ist ein wahrer Daniel Düsentrieb. Mit wenigen Handbewegungen komponiert er aus seiner Hängematte die Vorlesung, findet GPS-gesteuert seinen Hörsaal, verlässt sich auf sein Display im Brillenglas und eine Textiltechnik, die ihm den Computer auf den Leib schneidert. Dank biometrischer Daten kann er die Studentin in der dritthintersten Reihe mit Namen ansprechen, seine Antworten mit Suchmaschinen komplettieren und die elektronische
Agenda nach dem nächsten Date abfragen oder zu Hause die Badewanne vollaufen lassen. Seine intelligenten Klamotten vernetzen ihn mit dem Internet und der Gebäudetechnologie der Mensch-Maschinen-Interaktionen. Sein «Wearable Computing Lab» der ETH entwickelt miniaturisierte Wearables, in die Kleider eingearbeitete Mikrochips, sogenannte kontextsensitive Textilien, die mit einer externen Infrastruktur kommunizieren können. Wie alle Erfinder ist er restlos von der Nützlichkeit seiner Ideen überzeugt, und wie immer wird der Nutzen, abgesehen von den verschwiegenen militärischen Anwendungen, der Öffentlichkeit als medizinischer Fortschritt verkauft. Der Sport-BH, der Puls und Blutdruck misst, WC-Analyseaggregate für den Stuhlgang, Monitore und Smartphones für Risikopatienten, Sensoren für die Rehabilitation, Prothesen für Gelähmte, Telemedizin und Operationsroboter.

Niemand fragt, ob wir das wirklich wollen und brauchen, das Pervasive Computing und die neuen Sensoren. Der Markt eilt dem Konsumentenschutz davon. Was bleibt, sind die Risiken und der Elektroschrott. Die Technologien der E-Grains, der staubkorngrossen, elektronischen Fühler, verheissen ein digitales Schlaraffenland aus gefahrloser Wartung von Industrieanlagen, Verkehrstelematik und intelligenten Haushaltgegenständen. Was sie aber mit tödlicher Sicherheit ermöglichen, ist eine Welt der totalen Kontrolle, der elektronischen Fesseln, der «smart bombs», der neuen Cyberkriminalität, des beschleunigten Wandels unserer sozialen Strukturen und der zunehmenden Verwundbarkeit der Informationsgesellschaft. 